\title{
Herbivore-induced terpenoid emission in Medicago truncatula: concerted action of jasmonate, ethylene and calcium signaling
}

\author{
Gen-ichiro Arimura $\cdot$ Stefan Garms $\cdot$ Massimo Maffei $\cdot$ \\ Simone Bossi • Birgit Schulze $\cdot$ Margit Leitner $\cdot$ \\ Axel Mithöfer $\cdot$ Wilhelm Boland
}

Received: 30 March 2007 / Accepted: 12 September 2007 / Published online: 9 October 2007

(C) Springer-Verlag 2007

\begin{abstract}
Plant volatiles emitted by Medicago truncatula in response to feeding larvae of Spodoptera exigua are composed of a complex blend of terpenoids. The cDNAs of three terpene synthases (TPSs), which contribute to the blend of terpenoids, were cloned from M. truncatula. Their functional characterization proved MtTPS1 to be a $\beta$-caryophyllene synthase and MtTPS5 to be a multi-product sesquiterpene synthase. MtTPS3 encodes a bifunctional enzyme producing $(E)$-nerolidol and geranyllinalool (precursors of $\mathrm{C}_{11}$ and $\mathrm{C}_{16}$ homoterpenes) from different prenyl diphosphates serving as substrates. The addition of jasmonic acid (JA) induced expression of the TPS genes, but terpenoid emission was higher from plants treated with JA and the ethylene precursor 1-amino-cyclopropyl-1-carboxylic acid. Compared to infested wild-type M. truncatula plants, lower amounts of various sesquiterpenes and a $\mathrm{C}_{11^{-}}$ homoterpene were released from an ethylene-insensitive mutant $s k l$. This difference coincided with lower transcript levels of MtTPS5 and of 1-deoxy-D-xylulose-5-phosphate
\end{abstract}

Electronic supplementary material The online version of this article (doi:10.1007/s00425-007-0631-y) contains supplementary material, which is available to authorized users.

G.-i. Arimura $(\bowtie) \cdot$ S. Garms $\cdot$ B. Schulze $\cdot$ M. Leitner

A. Mithöfer $\cdot$ W. Boland $(\square)$

Department of Bioorganic Chemistry, Max Planck Institute

for Chemical Ecology, Hans-Knöll-Straße 8,

07745 Jena, Germany

e-mail: garimura@ice.mpg.de

W. Boland

e-mail: boland@ice.mpg.de

M. Maffei $\cdot$ S. Bossi

Department of Plant Biology and Centre of Excellence CEBIOVEM, University of Turin, Viale P.A. Mattioli 25, 10125 Turin, Italy synthase (MtDXS2) in the damaged $s k l$ leaves. Moreover, ethephon, an ethylene-releasing compound, modified the extent and mode of the herbivore-stimulated $\mathrm{Ca}^{2+}$ variations in the cytoplasm that is necessary for both JA and terpene biosynthesis. Thus, ethylene contributes to the herbivory-induced terpenoid biosynthesis at least twice: by modulating both early signaling events such as cytoplasmic $\mathrm{Ca}^{2+}$-influx and the downstream JA-dependent biosynthesis of terpenoids.

Keywords Calcium · Ethylene · Jasmonic acid · Medicago truncatula $\cdot$ Terpenoid

$\begin{array}{ll}\text { Abbreviations } \\ \text { ACC } & \text { 1-Aminocyclopropane-1-carboxylic acid } \\ \text { BAPTA } & \begin{array}{l}\text { 1,2-Bis-(2- aminophenoxy)ethane- } N, N, N^{\prime}, N^{\prime}- \\ \text { tetra acetic acid }\end{array} \\ \text { BAW } & \text { Beet armyworm } \\ \text { DMADP } & \text { Dimethylallyl diphosphate } \\ \text { DMNT } & \text { (E)-4,8-Dimethyl-1,3,7-nonatriene } \\ \left.{ }^{2} \mathrm{H}_{2}\right]-D O X & \text { 1-Deoxy-[5,5- }{ }^{\left.\mathrm{H}_{2}\right]-\mathrm{D}-x y l u l o s e} \\ \text { DXP } & \text { 1-Deoxy-D-xylulose-5-phosphate } \\ \text { DXS } & \text { DXP synthase } \\ \text { FDP } & \text { Farnesyl diphosphate } \\ \text { GDP } & \text { Geranyl diphosphate } \\ \text { GGDP } & \text { Geranylgeranyl diphosphate } \\ \text { HIPV } & \text { Herbivore-induced plant volatile } \\ \text { HMGR } & \text { 3-Hydroxy-3-methyl-glutaryl } \\ & \text { CoA reductase } \\ \text { IDP } & \text { Isopentenyl diphosphate } \\ \text { JA } & \text { Jasmonic acid } \\ \text { MEP } & \text { 2-C-methyl-D-erythritol 4-phosphate } \\ \text { MVA } & \text { Mevalonic acid } \\ \text { OPDA } & \text { 12-Oxophytodienoic acid } \\ \text { SPME } & \text { Solid-phase microextraction } \\ \end{array}$


STS Silver thiosulfate

TMTT $\quad(E, E)-4,8,12$-Trimethyltrideca-1,3,7, 11-tetraene

TPS

Terpene synthase

\section{Introduction}

Volatile terpenoids, the major products among the herbivoreinduced plant volatiles (HIPVs) in the legume Medicago truncatula, include monoterpenes $\left(\mathrm{C}_{10}\right)$, sesquiterpenes $\left(\mathrm{C}_{15}\right)$, and tetranor-terpenoids (homoterpenes, $\mathrm{C}_{11}$ or $\mathrm{C}_{16}$ ) (Leitner et al. 2005). The biosynthetic routes to terpenes are fed by either the mevalonate (MVA) pathway in the cytosol/endoplasmic reticulum or the 2-C-methyl-D-erythritol 4phosphate (MEP) pathway in the plastids; both pathways generate the five-carbon compound isopentenyl diphosphate (IDP) and its isomer dimethylallyl diphosphate (DMADP). Cytosolic and plastidic prenyltransferases, respectively, synthesize farnesyl diphosphate (FDP) as a substrate for sesquiterpenes and geranyl diphosphate (GDP) and geranylgeranyl diphosphate (GGDP) as substrates for mono- and diterpenes (Rodríguez-Concepción and Boronat 2002; Eisenreich et al. 2004). Both pathways can cross-talk by exchanging intermediates (e.g., IDP or GDP) (Bick and Lange 2003). Molecular diversity is further expanded by the terpene synthases (TPSs), which utilize the different prenyl diphosphates as substrates. The transcript levels of four genes for terpene synthases, TPSs $1-4$, are enhanced in M. truncatula leaves damaged by Spodoptera exigua (beet armyworm [BAW]) herbivory or treated with methyl jasmonate (Gomez et al. 2005). Terpenoid formation is generally assumed to be regulated on the transcript level of the TPS genes (Dudareva et al. 2003; McKay et al. 2003; SharonAsa et al. 2003; Arimura et al. 2004a), but the mode of regulation is often complex (Lücker et al. 2001; Aharoni et al. 2003; Arimura et al. 2004b; Aharoni et al. 2005) and needs to be studied individually with respect to the plant and the herbivore.

Till now, little has been known about early physiological events and the interaction between the signaling networks in plants that occur when herbivores feed. The interaction of various pathways in the networks is assumed to result in an integrated overall response that initiates the emission of a characteristic volatile pattern. Since the blends of HIPVs may vary with the attacking herbivore (De Moraes et al. 1998; Ozawa et al. 2000; Leitner et al. 2005), various components and cross-talk between the involved signaling pathways are thought to be responsible for the characteristic terpenoid blend (Engelberth et al. 2001; Schmelz et al. 2003b). Several oxylipin compounds (jasmonic acid (JA), its precursors, and related compounds) very likely act as master switches for herbivore-stimulated plant responses, activating distinct sets of defense genes leading to terpenoid formation (Arimura et al. 2004a; Ament et al. 2006). In addition, antagonistic or synergistic cross-reactions with other regulators, in particular involving ethylene and salicylic acid, seem to control and coordinate the formation of a characteristic blend of volatiles (Ozawa et al. 2000; Engelberth et al. 2001; Horiuchi et al. 2001; Schmelz et al. 2003b). Although ethylene is a well known modulator of processes of plant development and plant defense against biotic and abiotic stresses (Wang et al. 2002), only little is known about how ethylene affects the composition of herbivore-induced volatiles (Kahl et al. 2000; Schmelz et al. 2003a; Schmelz et al. 2003b). Using the model plant M. truncatula, we compared the ethylene-insensitive mutant sickle ( $(\mathrm{kl})$ with wild-type plants and showed that the ethylene signaling cascade modulates the intracellular $\mathrm{Ca}^{2+}$-level and interacts with JA signaling to generate a specific blend of terpenoids in response to feeding BAW larvae. In addition, three cDNAs encoding new terpene synthases, MtTPS1, MtTPS3, and MtTPS5, were cloned from M. truncatula and heterologously expressed for biochemical characterization.

\section{Materials and methods}

Plants and caterpillars

Plants of M. truncatula, wild-type cv. Jemalong (Pogue Agri Partners, Kenedy, TX, USA) and the ethylene-insensitive mutant $s k l$ ( $s k l l-1$ : isolated and backcrossed by the group of Dr. Douglas R. Cook, University of California, Davis) were grown in soil. Each plastic pot contained one or two plants and was kept in a growth chamber at $27^{\circ} \mathrm{C}$ (14 h light:10 h dark; relative humidity: 65\%) for 6-8 weeks. Plants without flowers were used for each treatment. Spodoptera exigua [beet armyworm (BAW)] larvae were reared on artificial diet in a plastic box $\left(22 \pm 1^{\circ} \mathrm{C} ; 14 \mathrm{~h}\right.$ light: $10 \mathrm{~h}$ dark) (Bergomaz and Boppré 1986). For BAW infestation, 5-6 third-instar larvae were placed on shoots of M. truncatula.

\section{Chemical treatment}

A solution containing JA (1 mM, pH 5.8-6.0), 1-aminocyclopropane-1-carboxylic acid (ACC, $1 \mathrm{mM}$; Sigma-Aldrich, St Louis, MO, USA), and/or silver thiosulfate (STS, $1 \mathrm{mM}$; Sigma-Aldrich) in $20 \mathrm{ml}$ of water was evenly sprayed onto intact plants growing in plastic pots. Lower concentrations of JA and ACC solutions (e.g. $0.3 \mathrm{mM}$ each) had little effect on induced volatiles in M. truncatula plants. For ethephon treatment, ethephon (10 mM; Sigma-Aldrich) in $100 \mu \mathrm{l}$ of $50 \mathrm{mM}$ MES buffer, $\mathrm{pH}$ 6.0, was applied to 
leaves of an intact $M$. truncatula plant. For inhibition experiments, the petioles of detached plantlets of $M$. truncatula were placed in glass vials containing aqueous solutions of fosmidomycin $(7 \mathrm{ml}, 100 \mu \mathrm{M}$; Molecular Probes, Eugene, OR, USA), or lovastatin (7 ml, $100 \mu \mathrm{M}$; A.G. Scientific, San Diego, CA, USA). Incubation experiments with labelled precursors were carried out by placing the plantlets in water $\left(7 \mathrm{ml}\right.$, containing 1-deoxy-[5,5- $\left.{ }^{2} \mathrm{H}_{2}\right]$-D-xylulose at $1 \mathrm{mg} / \mathrm{ml}$ ). Controls were also placed in water. After preincubation with the labelled precursor or the inhibitors for $2 \mathrm{~h}$, either JA + ACC (1 mM each) in $20 \mathrm{ml}$ of water were sprayed evenly onto the plants or 5-6 larvae were placed on the plants. For headspace analysis, the plants were transferred $2 \mathrm{~h}$ after JA + ACC treatment into a glass cabinet for volatile collection (see below). Each treatment started at 10:00. During treatments, temperatures were kept constant at $22 \pm 1^{\circ} \mathrm{C}$, and the photoperiod was $14 \mathrm{~h}$ light $(6,000$ lux): $10 \mathrm{~h}$ dark. The light period extended from 07:00 to 21:00.

\section{Analysis of volatiles}

For headspace analysis, a potted plant or a plantlet in a glass vial was enclosed with or without caterpillars in glass containers (2.5 1). The emitted volatiles were trapped onto charcoal traps (1.5 mg of charcoal, CLSA-Filter, Le Ruisseau de Montbrun, Daumazan sur Arize, France) while air circulated for 24 or $48 \mathrm{~h}$. The collected volatiles were eluted with dichloromethane $(2 \times 20 \mu \mathrm{l})$ containing $n$-bromodecane (100 ng $\mu \mathrm{l}^{-1}$ ) as an internal standard. Samples were analyzed on a ThermoQuest/Finnigan TRACE GC 2000 with a TRACE MS (Manchester, UK) equipped with an $\mathrm{EC}^{\mathrm{TM}}-5$ capillary column $(0.25 \mathrm{~mm}$ i.d. $\times 15 \mathrm{~m}$ with $0.25-\mathrm{mm}$ film, Alltech, Deerfield, IL, USA). Injection volume: $1 \mu \mathrm{l}$; split $1: 100 ; 220^{\circ} \mathrm{C}$. Ionization energy: $70 \mathrm{eV}$. Compounds were eluted under programmed conditions starting from $40^{\circ} \mathrm{C}(2-$ min hold) and ramped up at $10^{\circ} \mathrm{C} \mathrm{min}^{-1}$ to $200^{\circ} \mathrm{C}$ followed by $30^{\circ} \mathrm{C} \mathrm{min}^{-1}$ to $280^{\circ} \mathrm{C}$, which was held for $1 \mathrm{~min}$ prior to cooling. Helium at a flow rate of $1.5 \mathrm{ml} \mathrm{min}{ }^{-1}$ served as a carrier gas. The headspace volatiles were identified by comparing their mass spectra and Kovàts indices with authentic references on two columns of different polarity (Leitner et al. 2005). Absolute quantification of the volatiles was not possible due to the limited availability of authentic standards and partly overlapping peak areas of germacrene D $\mathbf{1 6}$ and an unknown sesquiterpene 17. Relative peak areas from integration of the reconstructed ion chromatogram were used without additional calibration.

\section{Analysis of oxylipins}

Leaves $(0.5 \mathrm{~g})$ were homogenized with an Ultra-Turrax ${ }^{\circledR}$ T25 Basic (Ika, Staufen, Germany) on ice for $5 \mathrm{~min}$ in methanol $(8 \mathrm{ml})$ containing $0.03 \%$ BHT and $0.03 \mathrm{M}$ pentafluorobenzyl hydroxylamine hydrochloride (PFBHA, Sigma-Aldrich) (Schulze et al. 2006). $\left[{ }^{2} \mathrm{H}_{2}\right]-\mathrm{JA}$ (150 ng) and $\left[{ }^{2} \mathrm{H}_{2}\right]$-12-oxophytodienoic acid $(250 \mathrm{ng})$ were added as internal standards. The methanol extract was shaken for derivatisation at room temperature for $2 \mathrm{~h}$, and $4.0 \mathrm{ml}$ diluted $\mathrm{HCl}(\mathrm{pH}=3)$ were added. The methanol/water phase was carefully extracted with hexane $(3 \times 10 \mathrm{ml})$, and after centrifugation, the hexane layers were collected and combined. The hexane fraction was passed through a Chromabond $\mathrm{NH}_{2}$ cartridge (3 ml/500 mg, MachereyNagel, Düren, Germany) preconditioned with methanol $(5 \mathrm{ml})$ and hexane $(5 \mathrm{ml})$, washed with the solvent mixture of 2-propanol/dichloromethane $(2: 1 \mathrm{v} / \mathrm{v}, 5 \mathrm{ml})$, and eluted with diethyl ether/formic acid $(98: 2,10 \mathrm{ml})$. The solvents were evaporated under argon, and the remaining crude acids were esterified with an ethereal solution of diazomethane $(1 \mathrm{ml})$ at room temperature for $5 \mathrm{~min}$. After evaporation of the solvent, the sample was dissolved in $30 \mu \mathrm{l}$ dichloromethane and analyzed by gas chromatographymass spectrometry (GC-MS) as described (Schulze et al. 2006).

\section{Cytoplasmic $\mathrm{Ca}^{2+}$ concentration}

A solution of Fluo-3 AM (acetoxy-methyl ester of Fluo-3, $5 \mu \mathrm{M}$, Fluka, Buchs, Switzerland), $0.5 \mathrm{mM}$ calcium sulphate, and 2.5 $\mu \mathrm{M}$ DCMU [3-(3',4'-dichlorphenyl)-1,1dimethylurea] in $50 \mathrm{mM}$ MES buffer, $\mathrm{pH}$ 6.0, was used for initial treatment of leaves of an intact $M$. truncatula plant as previously described (Maffei et al. 2004). A leaf was cut once by a razor blade in order to allow the dye to enter the tissues. One hour after treatment with Fluo-3 AM, the leaf was fixed on an Olympus FLUOview confocal laser scanning microscope stative without detaching it from the plant. The microscope was operated with a Krypton/ Argon laser at 488 and $568 \mathrm{~nm}$ wavelengths: the first wavelength excited the Fluo-3 dye emitting green light, while the second excited mostly chloroplasts emitting a red fluorescence. Images generated by the FluoView software were analyzed using the NIH Image J software described earlier (Maffei et al. 2004). Aequorin-dependent luminescence was determined according to (Maffei et al. 2006).

For high resolution subcellular $\mathrm{Ca}^{2+}$ localization, after treatment with Fluo-3 AM for $1 \mathrm{~h}$, leaves were mounted on a Leica TCS SP2 multiband confocal laser-scanning microscope stative without separating the leaf from the plant. Scannings were recorded using the HCX PL APO 63x/1.20 W Corr/0.17CS objective. The microscope was operated with a Laser Ar (458 nm/5 mW; $476 \mathrm{~nm} / 5 \mathrm{~mW} ; 488 \mathrm{~nm} /$ $20 \mathrm{~mW}$; $514 \mathrm{~nm} / 20 \mathrm{~mW}$ ), a Laser HeNe $543 \mathrm{~nm} / 1.2 \mathrm{~mW}$, and a Laser HeNe $633 \mathrm{~nm} / 10 \mathrm{~mW}$. 


\section{Real-time PCR}

Total RNA was isolated from leaf tissues using the Concert Plant RNA Reagent (Invitrogen, Carlsbad, CA, USA) following the manufacturer's protocol. Total RNA was purified to eliminate genomic DNA using the Qiagen RNeasy Plant RNA kit and the RNase-Free DNase Set (Qiagen, Hilden, Germany). First-strand cDNA was synthesized using the SuperScript III Reverse Transcriptase (RT) (Invitrogen), oligo(dT) $)_{12-18}$ primer, and $2 \mu \mathrm{g}$ of total RNA at $50^{\circ} \mathrm{C}$ for $50 \mathrm{~min}$. Primers for real-time polymerase chain reaction (PCR) were designed using the Primer 3 Software (http:// frodo.wi.mit.edu/cgi-bin/primer3/primer3_www.cgi) for a length of the resulting PCR product of approximately $200 \mathrm{bp}$. Primers were designed using partial DNA sequences obtained from the TIGR $M$. truncatula expressed sequence tag (EST) database: (http://www.tigr.org/tigrscripts/tgi/T_index.cgi?species=medicago) and GenBank. The real-time PCR was done on a Mx3000P Real-Time PCR System (Stratagene, La Jolla, CA, USA). The process was performed with $25 \mu \mathrm{l}$ of reaction mixture containing $12.5 \mu$ of $2 \times$ Brilliant SYBR Green QPCR Master Mix (Stratagene), cDNA ( $1 \mu \mathrm{l}$ from $20 \mu \mathrm{l}$ of each RT product pool), $100 \mathrm{nM}$ primers, and $30 \mathrm{mM} \mathrm{ROX}$ as a passive reference dye. The following protocol was followed: initial polymerase activation: $10 \mathrm{~min}$ at $95^{\circ} \mathrm{C} ; 40$ cycles of $30 \mathrm{~s}$ at $95^{\circ} \mathrm{C}, 60 \mathrm{~s}$ at $55^{\circ} \mathrm{C}$, and $30 \mathrm{~s}$ at $72^{\circ} \mathrm{C}$. PCR conditions were determined by comparing threshold values in dilution series of the RT product, followed by non-RT template control and non-template control for each primer pair. Relative RNA levels were calibrated and normalized with the level of actin mRNA (EST annotation no. AA660796).

Primers used for real-time PCR are: actin, 5'-TAC CCC ATT GAG CAC GGT AT-3' and 5'-ATA CAT GGC AGG CAC ATT GA-3'; MtDXS1, 5'-AGC CGT TCA GAA CTG TTT GG-3' and 5'-ATC AAG GGC CAT GAA CTG AG-3'; MtDXS2, 5'-ACA CCG GTG GTC ATC TTA GC$3^{\prime}$ and 5'-CAT CCC TTT TGG GAA AAC CT-3'; MtHMGR1, 5'-GCC GTA AAT GAT GGG AGA GA-3' and 5'-GCC AGC TAA AAC AGC TCC AG-3'; MtTPS1, $5^{\prime}$-TGC TCC AAC CCC TAA TGC TA-3' and 5'-CCT CGA AGG GTT TGT GAC AT-3'; MtTPS2, 5' -TGT CGC AAA AAC AGA TGA GC-3' and 5'-ATC CTT GTT GCC TCA ACA CC-3'; MtTPS3, 5' -TGC AAT CAC AAA ATG CAA CA-3' and 5'-GAT CTG GGA GAC ATG CCA TT-3'; MtTPS5, 5'-CCG ATG CCT TTT ATG ACT CG-3' and $5^{\prime}$-TGT GCC ACT TGG ATT TTT CA-3'. The EST annotation numbers are: actin, AA660796; MtHMGR1, TC102857. GenBank accession numbers are: MtDXS1, AJ430047; MtDXS2, AJ430048; MtTPS1 AY763425; MtTPS2, AY766250; MtTPS3, AY766249; MtTPS5, DQ188184.
cDNA cloning and TPS enzyme assay

Cloning 3 '-end of MtTPS5 cDNAs was accomplished by rapid amplification of cDNA ends (RACE PCR) using total RNA and the First Choice RLM-RACE Kit (Ambion, Austin, TX, USA) following the manufacturer's protocol. For functional identification, cDNAs were amplified by PCR using $P f u$ DNA Polymerase (Promega, Madison, WI, USA) with a set of primers for an open reading frame of MtTPS1 MtTPS3, or MtTPS5. The cDNA was subcloned into the pHis8-3 expression vector (Jez et al. 2000). The recombinant vectors were transformed into Escherichia coli BL21-CodonPlus(DE3). The bacterial strain was grown to $A_{600}=0.5$ at $37^{\circ} \mathrm{C}$ in $5 \mathrm{ml}$ of $\mathrm{LB}$ medium with kanamycin at $50 \mu \mathrm{g} / \mathrm{ml}$. Cultures were induced with $1 \mathrm{mM}$ isopropyl 1-thio- $\beta$-D-galactopyranoside (IPTG) and held overnight at $16^{\circ} \mathrm{C}$ while being shaken at $200 \mathrm{rpm}$. Cells were pelleted by centrifugation and resuspended in $1 \mathrm{ml}$ TPS buffer (25 mM HEPES, $\mathrm{pH}=7.3,12.5 \mathrm{mM} \mathrm{MgCl}_{2}$, $0.25 \mathrm{mM} \quad \mathrm{MnCl}_{2}, 0.25 \mathrm{mM} \mathrm{NaWO}_{4}, 0.125 \mathrm{mM} \mathrm{NaF}$, $5 \mathrm{mM}$ DTT, $10 \%$ glycerol). Resuspended cells were broken by sonication. Cell extracts were cleared by centrifugation and assayed for TPS activity with $50 \mu \mathrm{M}$ GDP (Echelon Biosciences Incorporated, Salt Lake City, UT, USA), FDP (Echelon Biosciences Incorporated) or GGDP (Sigma-Aldrich). The assay mixture $(1 \mathrm{ml})$ was covered with $1 \mathrm{ml}$ of pentane to trap volatile products. After incubation at $30^{\circ} \mathrm{C}$ for $1 \mathrm{~h}$, the pentane layer was transferred to a glass vial and concentrated to $\sim 150 \mu$ l under a gentle stream of $\mathrm{N}_{2}$. Alternatively, the headspace of the assay mixture was analyzed using solid-phase microextraction (SPME, Supelco Inc., Bellefonte, PA, USA). Extracts of $E$. coli transformed with expression vectors without the TPS gene were used as controls following the above procedure. The enzymatic products were analyzed by GCMS and identified by comparing their mass spectra and Kovàts indices with authentic references as described above.

Stereochemistry of $(E)$-nerolidol was analyzed by a Hewlett-Packard 5980 gas chromatograph coupled to a Hewlett-Packard 5917A quadrupole type mass selective detector (70 eV; scan range: 45-400 amu) equipped with a capillary column coated with heptakis-(2,6-di- $O$-methyl-3$O$-pentyl)- $\beta$-cyclodextrin $(0.25 \mathrm{~mm} \times 25 \mathrm{~m}, 0.25 \mu \mathrm{m}$ film, Macherey Nagel, Düren, Germany). Samples (1 $\mu \mathrm{l})$ were injected splitless at $230^{\circ} \mathrm{C}$ with a column flow of $1 \mathrm{ml}$ $\min ^{-1}(\mathrm{He})$. Elution was programmed from 50 to $160^{\circ} \mathrm{C}$ (10 min) at $2.3^{\circ} \mathrm{C} \mathrm{min}^{-1}$ and then to $210^{\circ} \mathrm{C}$ at $15^{\circ} \mathrm{C} \mathrm{min} \mathrm{min}^{-1}$ $(2 \mathrm{~min})$. The elution order of $(3 S)-(E)$-nerolidol and $(3 R)$ $(E)$-nerolidol was determined with a racemic and a chiral reference according to (Degenhardt and Gershenzon 2000). 


\section{Results}

HIPV biosynthesis in $M$. truncatula WT and the ethylene-insensitive mutant $s k l$

The plant hormone ethylene is generally involved in processes of plant development and plant defense against biotic and abiotic stresses (Wang et al. 2002). In genetic studies using $M$. truncatula, the ethylene-insensitive mutant $s k l$ displayed a supernodulation phenotype (Penmetsa and Cook 1997; Oldroyd et al. 2001), demonstrating that ethylene is an essential element in the interaction between root and rhizobia. We used $s k l$ to study whether and how ethylene is involved in the signaling cascades leading to characteristic blends of HIPVs. To obtain sufficient amounts of HIPV from herbivore-damaged plants, volatiles were continuously collected for $48 \mathrm{~h}$ after exposure to BAW feeding (Fig. 1a). Wild-type (WT) plants exposed to BAW larvae released a blend of volatiles comprising fatty acid-derived compounds, sesquiterpenes, and two homoterpenes. On the other hand, BAW-damaged skl plants released significantly lower amounts of certain sesquiterpenes ( $\alpha$-copaene, germacrene D along with an unidentified sesquiterpene (I), $\alpha$-muurolene, $(E)$-nerolidol, and $\beta$-himachalol), and a homoterpene $((E)$-4,8-dimethyl-1,3,7-nonatriene, DMNT). The monoterpene limonene was constitutively released only from $s k l$ plants but not from WT; after BAW damage, the emission of limonene increased (Fig. 1a, compound 3).

Next, we investigated transcript levels of genes of early and late steps in terpenoid biosynthesis in BAW-damaged WT and $s k l$ by quantitative RT-PCR analysis (Fig. 1b). The transcript levels of putative terpene synthases MtTPS2 and MtTPS3 (Gomez et al. 2005) were induced at similar levels in WT and $s k l$ plants throughout the entire period of BAW damage, whereas the transcript levels of MtTPS1 (Gomez et al. 2005) and the gene encoding 1-deoxy-D-xylulose-5phosphate (DXP) synthase (MtDXS2) involved in the MEP pathway (Walter et al. 2002) were initially lower in $s k l$ plants after exposure to BAW feeding $(2 \mathrm{~h}, P=0.08$ and $P<0.05$, respectively, ANOVA). A putative sesqui-TPS gene (MtTPS5) mined from the TIGR M. truncatula EST database was only up-regulated in WT plants after $24 \mathrm{~h}$. Unlike MtDXS2, the transcript levels of MtDXS1 and 3-hydroxy-3-methylglutaryl-CoA reductase (MtHMGRl), two other genes of early terpenoid biosynthesis, were identical in both BAW-damaged WT and skl plants.

\section{Product spectrum of MtTPSs}

MtTPS1 and MtTPS3 had been predicted to represent sesqui-TPS and mono-/di-TPS, due to both their sequence homology to TPSs from other plants and their localization
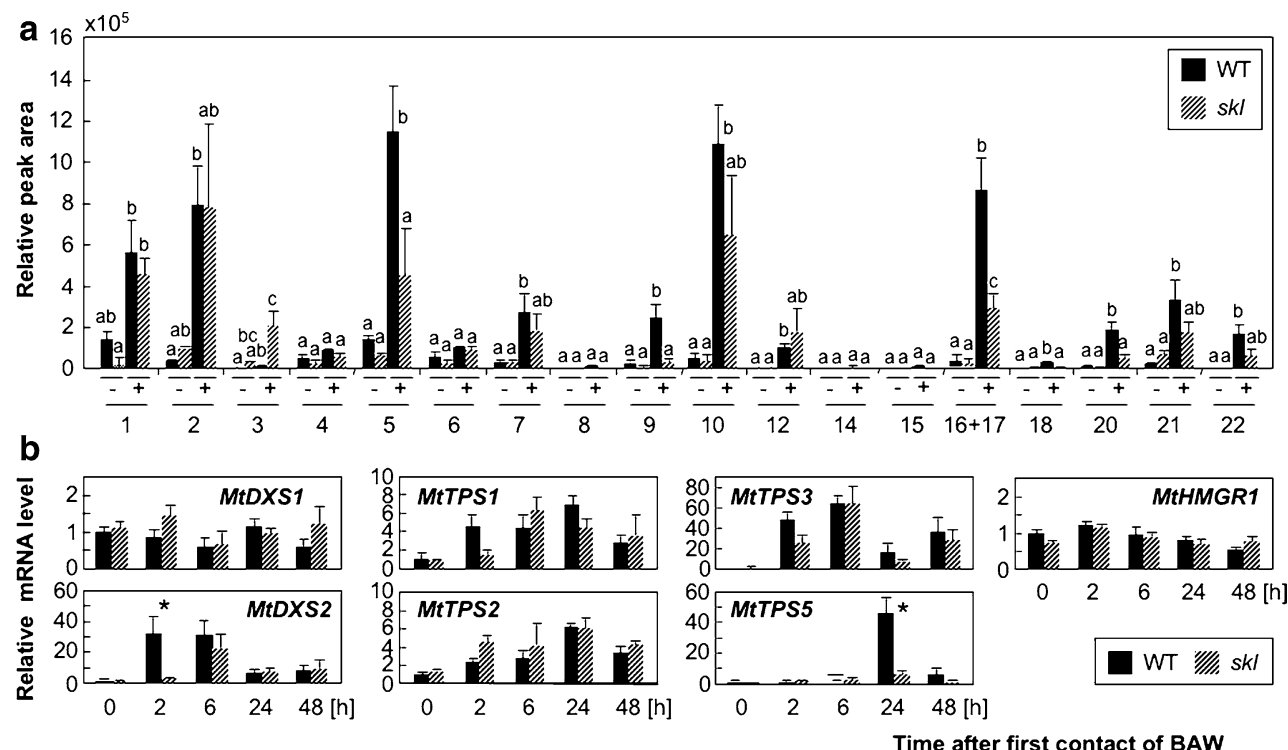

Fig. 1 Volatile profiles and mRNA levels in wild-type (WT) and in $s k l$ plants. a Volatiles emitted from WT and from $s k l$ plants damaged by beet armyworms (BAW); (+) with larvae, (-) without larvae for $48 \mathrm{~h}$. Emission is presented as relative peak area $(\mathrm{g} \text { shoot tissue })^{-1}$ collected over a 48 -h period $+\operatorname{SE}(n=4)$. Means followed by small letters for each set of volatiles that are significantly different according to Scheffe's test $(P<0.05) .1$ 1-octene-3-ol, $2(Z)$-3-hexenyl acetate, 3 limonene, $4 n$-nonanal, 5 DMNT, $6 n$-decanal, 7 (+)-cyclosativene, $8 \alpha$-ylangene, $9 \alpha$-copaene, $10 \beta$-caryophyllene, $12 \alpha$-himalachene, 14 allo-aromadendrene, $15 \gamma$-muurolene, 16 germacrene D, 17 unidentified sesquiterpene (I), $18 \alpha$-muurolene, 20 (E)-nerolidol, 21 TMTT, $22 \beta$-himachalol. Relative peak areas from integration of the reconstructed ion chromatogram were used without additional calibration. Peak areas of germacrene D and unidentified sesquiterpene $(16+17)$ were determined as the sum due to insufficient separation. b Relative mRNA levels for genes involved in terpenoid biosynthesis in BAW-infested leaves. Data represent the mean $+\operatorname{SE}(n=4)$. $* P<0.05$ (ANOVA). DXS DXP synthase, HMGR HMG-CoA reductase, TPS terpene synthase 
in cytosol and plastids, respectively (Gomez et al. 2005). Protein extracts from transformed bacterial cultures [E. coli strain BL21-CodonPlus(DE3)] containing and expressing the full-length coding region of either MtTPS1 or MtTPS3, were assayed with FDP as substrate. The products of MtTPS1 were identified as $\beta$-caryophyllene (92\% of total product) and $\alpha$-humulene ( $8 \%$ of total product, Fig. $2 a$ ). Although MtTPS3 has previously been predicted to represent a mono-/di-TPS (Gomez et al. 2005), the recombinant MtTPS3 generated $(E)$-nerolidol as a single product when supplied with FDP as substrate (Fig. 2a). A control extract prepared from the BL21-CodonPlus(DE3) strain transformed with a plasmid without the TPS cDNA insert did not produce significant amount of $(E)$-nerolidol $(0.6 \%$ residual production). The MtTPS3 product was identified as pure $(3 S)-(E)$-nerolidol by $\mathrm{GC}$ on a chiral $\beta$-cyclodextrin column (Fig. 2b).

In order to ensure the functional involvement of MtTPS5 in the HIPV biosynthesis, we determined a full-length cDNA sequence for the MtTPS5 from M. truncatula (GenBank accession no. DQ188184). The deduced protein sequence of MtTPS5 encodes a predicted protein of 553 amino acids that shares 55\% identity and $74 \%$ similarity with MtTPS1 and 49\% identity and 70\% similarity with poplar (Populus trichocarpa $\times$ deltoides) germacrene D synthase (AY438099). A functional assay with FDP as substrate resulted in the production of 11 sesquiterpene hydrocarbons: $\alpha$-cubebene, $\alpha$-copaene, $\beta$-cubebene, $\beta$ caryophyllene, $\beta$-copaene, $\alpha$-humulene, allo-aromadendrene, $\gamma$-muurolene, germacrene $\mathrm{D}, \alpha$-muurolene, $\delta$-cadinene, and four sesquiterpene alcohols, e.g., (-)-cubebol (Fig. 2a). All compounds-except two sesquiterpene alcohols-were unambiguously identified by their Kováts indices and mass fragmentation patterns using combined GC-MS and authentic standards. Interestingly, the alcohols were not detected in the profile of plant volatiles (see Fig. 1a). With GDP as substrate the recombinant MtTPSs yielded traces of monoterpenes (Fig. 3a). For example, recombinant MtTPS1 and MtTPS5 synthesized the monoterpenes myrcene, limonene, $\beta$-ocimene, terpinolene, $\alpha$-terpineol, and three unidentified monoterpenes at about $5 \%$ of the rate of sesquiterpene products. MtTPS3 produced linalool at $5 \%$ of the rate of $(E)$-nerolidol. Using GGDP both TPS failed to produce diterpenoids (Fig. 3b). In contrast, the recombinant MtTPS3 generated the diterpene geranyllinalool when supplied with GGDP (ca. 65\% of the rate of $(E)$-nerolidol). The oxidative degradation of $(E)$-nerolidol and geranyllinalool generates the homoterpenes DMNT and $(E, E)$-4,8,12-trimethyltrideca-1,3,7,11-tetraene (TMTT) (Donath and Boland 1994), both of which have been found in the blend of
Fig. 2 a Sesquiterpenes formed by the assay of the recombinant MtTPS enzymes with FDP as substrate. An assay of the extract prepared from the BL21-CodonPlus(DE3) strain transformed with a plasmid without the TPS cDNA insert serves as control. b Stereochemistry of $(E)$-nerolidol was identified by comparing the retention time with that of authentic standards of $(3 R)-(E)$-nerolidol and $(3 S)-(E)-$ nerolidol using GC-MS. Also shown is the sesquiterpene product formed by recombinant MtTPS3 enzyme in vitro
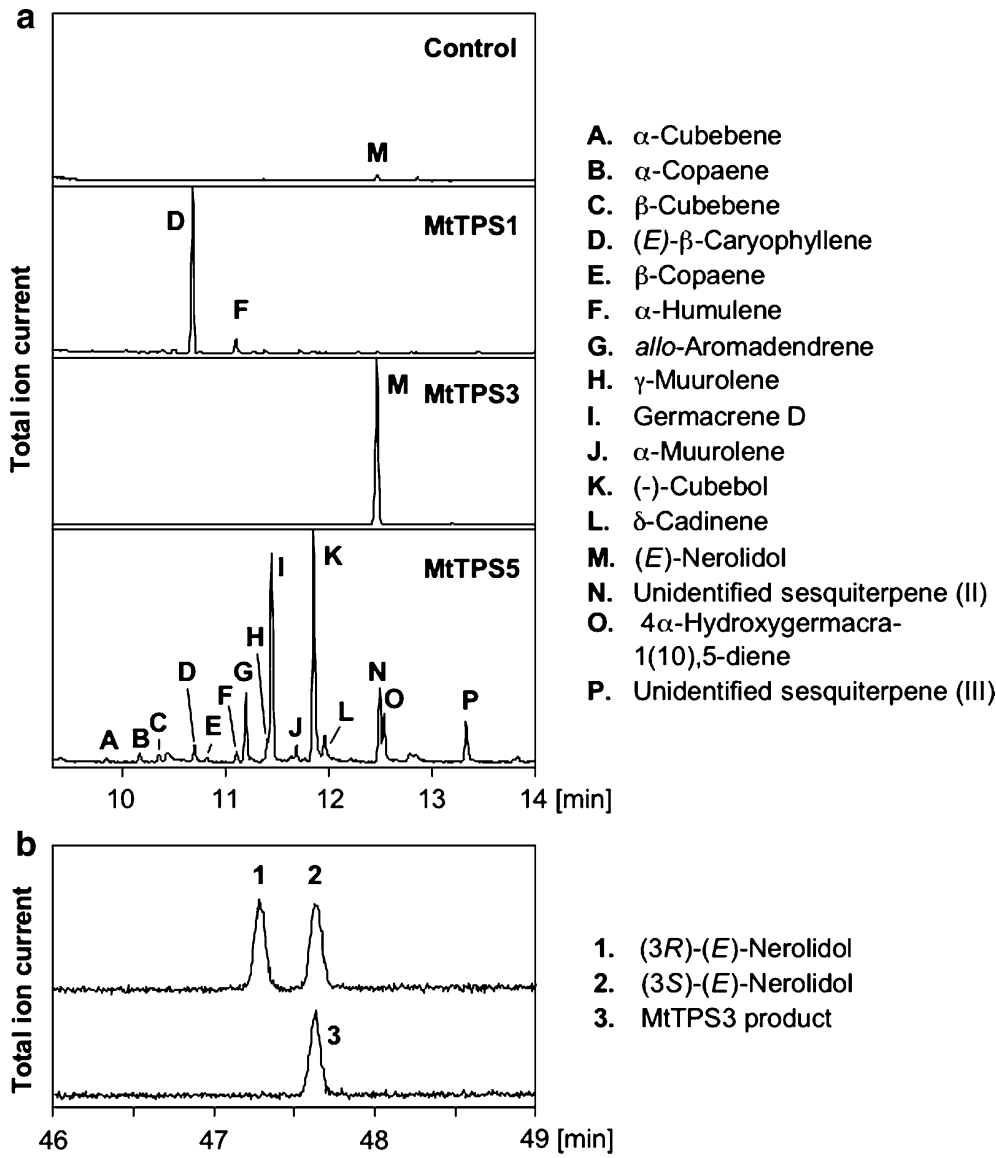
Fig. 3 Mono- and diterpenes formed by the assay of the recombinant MtTPS enzymes with GDP (a) and GGDP (b) as substrates, respectively. The GDPand GGDP-derived products trapped by SPME and pentene, respectively, are illustrated. Assays of the extract prepared from the BL21-CodonPlus(DE3) strain transformed with a plasmid without the TPS cDNA insert serve as control
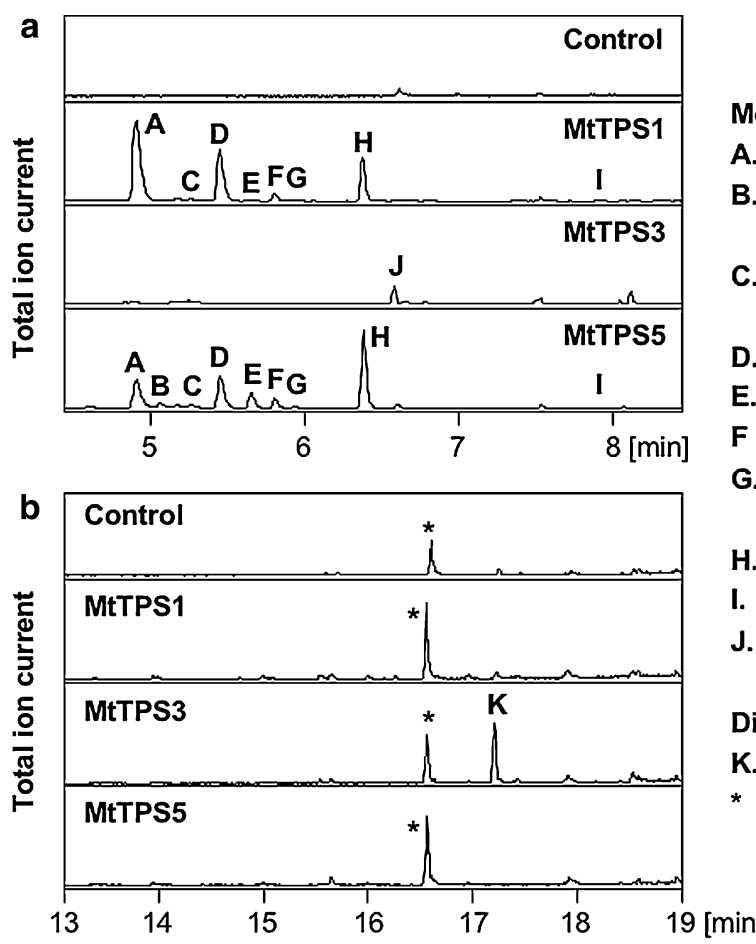

Monoterpene

A. Myrcene

B. Unidentified monoterpene (I)

C. Unidentified monoterpene (II)

D. Limonene

E. (Z)- $\beta$-Ocimene

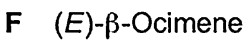

G. Unidentified monoterpene (III)

H. Terpinolene

I. $\alpha$-Terpineol

J. Linalool

Diterpene

K. Geranyllinalool

* Softener
HIPVs from BAW-damaged WT and $s k l$ plants (see Fig. 1a).

Synergistic effects of jasmonic acid and ACC on terpenoid biosynthesis

The endogenous levels of JA in WT and $s k l$ plants increased and reached a maximum $24 \mathrm{~h}$ after being exposed to feeding BAW larvae, but the differences between the two plants were not significant (Fig. S1 of electronic supplementary material). However, the composition of terpenoids depends on the concerted action of various signals, among which JA and ethylene are very likely important factors controlling TPS gene expression. To study the synergistic interaction between JA and ethylene signaling in WT leaf terpenoid biosynthesis, solutions of JA, ACC (precursor of ethylene), or JA + ACC were applied. Compared with the control, after $2 \mathrm{~h}$ JA induced MtDXS2, MtTPS1, and MtTPS3 transcripts, but after $24 \mathrm{~h}$ only MtTPS1 and MtTPS3 remained induced (Fig. 4). Treatment with ACC alone did not result in gene induction. Unlike the individual application of JA or ACC, their combined addition $(\mathrm{JA}+\mathrm{ACC})$ resulted in a significant increase of the transcript level of MtDXS2 after $2 \mathrm{~h}$ and of MtTPS5 after $24 \mathrm{~h}$. The mRNA levels of MtHMGRI and MtDXS1 were never significantly enhanced (Fig. 4).

Since after $2 \mathrm{~h}$ the emission of volatiles was not detectable, the effect of JA, ACC and JA + ACC was investigated with volatiles that were continuously collected for $24 \mathrm{~h}$ after spraying the solution on WT M. truncatula plants

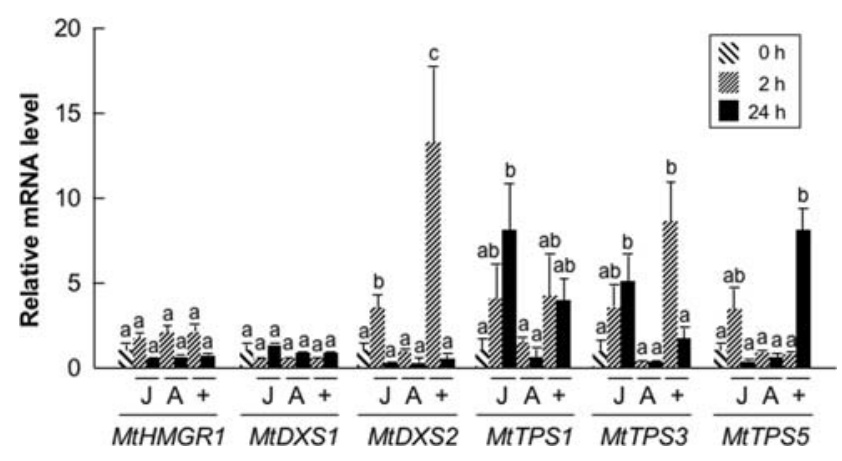

Fig. 4 Effect of exogenous application of JA and ACC on relative mRNA levels for genes involved in the biosynthesis of terpenoids in WT leaves. Treatments: JA (J), the ethylene precursor ACC (A), or both (+) for 2 and $24 \mathrm{~h}$ (mean + SE, $n=3-4)$. Means followed by small letters for each set of volatiles that are significantly different according to Scheffe's test $(P<0.05)$

(Fig. 5). $\beta$-Caryophyllene, the product of MtTPS1, was induced by $\mathrm{JA}$ and $\mathrm{JA}+\mathrm{ACC}$, whereas $(E)$-nerolidol and, to a greater extent, its degradation product DMNT resulting from MtTPS3 activity were induced by JA (DMNT) and by $\mathrm{JA}+\mathrm{ACC}$ (DMNT and $(E)$-nerolidol). The products of MtTPS5 were found mainly after stimulation by JA + ACC. The stimulatory effect of ethylene on the JA-linked terpenoid biosynthesis was also demonstrated by adding STS, a known inhibitor of ethylene perception (Veen 1983). Application of JA + ACC + STS to WT plants reduced the emission of JA + ACC-induced DMNT and other sesquiterpenes. 


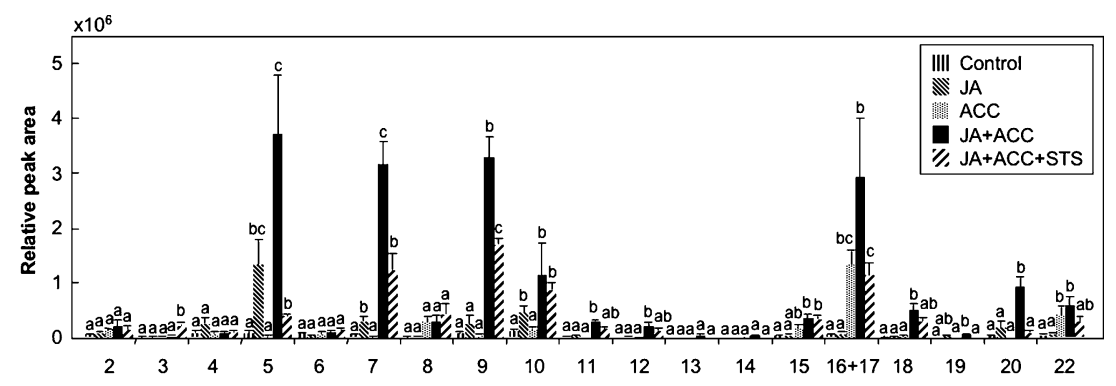

Fig. 5 Effect of exogenous application of JA and ACC: volatiles released from WT plants treated with water (control), JA, ACC, $\mathrm{JA}+\mathrm{ACC}$, or JA + ACC + STS for $24 \mathrm{~h}$. Emission is presented as relative peak area ( $\mathrm{g}$ shoot tissue) ${ }^{-1}$ collected over a $24-\mathrm{h}$ period $+\mathrm{SE}$ $(n=4-5)$. Relative peak areas from integration of the reconstructed ion chromatogram were used without additional calibration. Peak areas of germacrene $\mathrm{D}$ and unidentified sesquiterpene $(16+17)$ were determined as the sum due to insufficient separation. Means followed by

Pathway allocation of sesquiterpene biosynthesis in BAW-treated plants

In addition to the cytosolic MEV pathway, the plastidial MEP pathway, represented by the strongly responding $M t D X S 2$, should be the main source for herbivore-induced sesquiterpene biosynthesis. Therefore, we analyzed the contribution of the MEP pathway to sesquiterpene biosynthesis by administering 1-deoxy-[5,5- $\left.{ }^{2} \mathrm{H}_{2}\right]$-D-xylulose $\left(\left[{ }^{2} \mathrm{H}_{2}\right]\right.$-DOX (Meyer et al. 2004)) to WT M. truncatula followed by induction with JA + ACC (Fig. 6a). The emitted sesquiterpenes $\alpha$-copaene, $\beta$-caryophyllene, and $(E)$-nerolidol displayed a degree of labelling between 65 and $79 \%$, demonstrating that the MEP pathway contributed substantially to their biosynthesis. On average, the degree of deuterium labelling was between 65 and $80 \%$ for all sesquiterpenes; only the degree of labelling of DMNT was lower ( $40 \%$ of the total, Fig. $6 \mathrm{a}$ ). In addition, the relative contribution of the two pathways to the sesqui- and homoterpene biosyntheses was further tested by treating the plantlets with specific inhibitors (Fig. 6b). The MEP pathway was blocked by the application of fosmidomycin, a specific inhibitor of the DXP-reductoisomerase (Kuzuyama et al. 1998), and the MVA pathway was selectively inhibited by lovastatin, an inhibitor of HMGR (Bach and Lichtenthaler 1982). As shown in Fig. 6b, both pathways contribute to sesquiterpene biosynthesis, since both inhibitors suppressed the formation of the sesquiterpenes to a characteristic extent.

Impact of ethylene on $\mathrm{Ca}^{2+}$ signaling

Besides JA and ethylene, other factors might be involved in controlling terpenoid biosynthesis. In Lima bean, the first event after leaf feeding is an influx of $\mathrm{Ca}^{2+}$ in the damaged area (Maffei et al. 2004). Moreover, applying the $\mathrm{Ca}^{2+}$-chelator BAPTA to Lima bean leaves has been shown to small letters for each set of volatiles are significantly different according to Scheffe's test $(P<0.05) .2(Z)$-3-hexenyl acetate, 3 limonene, $4 n$-nonanal, 5 DMNT, $6 n$-decanal, 7 (+)-cyclosativene, $8 \alpha$-ylangene, $9 \alpha$-copaene, $10 \beta$-caryophyllene, $11 \beta$-copaene, $12 \alpha$-himalachene, 13 $\alpha$-humulene, 14 allo-aromadendrene, $15 \gamma$-muurolene, 16 germacrene $\mathrm{D}, 17$ unidentified sesquiterpene (I), $18 \alpha$-muurolene, $19 \delta$-cadinene, $20(E)$-nerolidol, $22 \beta$-himachalol
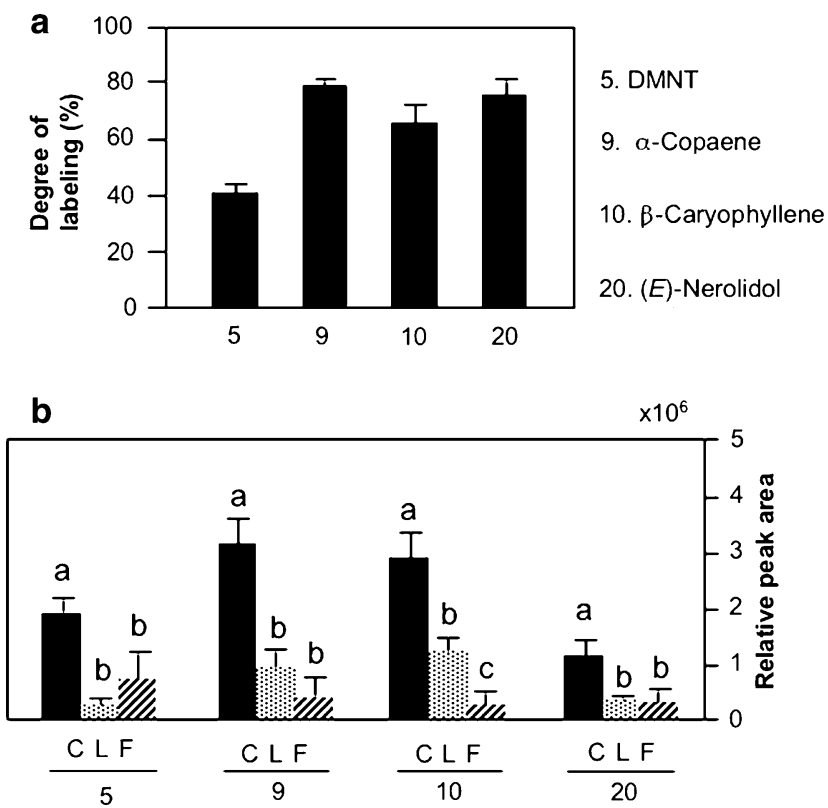

Fig. 6 Pathway allocation in terpenoid biosynthesis in JA + ACC induced M. truncatula. a Degree of labelling of terpenoids emitted from JA + ACC-induced plants pre-treated with $\left[{ }^{2} \mathrm{H}_{2}\right]$-DOX. b Volatiles from JA + ACC-induced plants after pre-treatment with lovastatin (L), fosmidomycin $(\mathrm{F})$, or water $(\mathrm{C})$. Emission is presented as relative peak area $(\mathrm{g} \text { shoot tissue })^{-1}$ collected over a 24 -h period $+\mathrm{SE}(n=3-4)$. Relative peak areas from integration of the reconstructed ion chromatogram were used without additional calibration. Means followed by small letters for each set of volatiles are significantly different according to Scheffe's test $(P<0.05)$

suppress the herbivore-induced transcript of defenserelated genes involved in sesquiterpene biosynthesis (Arimura et al. 2000). Therefore, we next studied the impact of JA and ethylene on the level of $\mathrm{Ca}^{2+}$ in $M$. truncatula WT and $s k l$ leaves after damage with BAW. The membranepermeable Fluo-3 AM $\left[\mathrm{Ca}^{2+}\right.$-sensitive fluorescent probe] was applied to leaf tissue of $M$. truncatula, and showed 
cytoplasmic subcellular localization (Fig. 7a). While the treatment of WT leaves with JA showed no visible effect on intracellular $\mathrm{Ca}^{2+}$ variation (Fig. S2 of electronic supplementary material). On the other hand, ethephon, a releasing source of ethylene (Edelmann et al. 2002), had a strong impact on the $\mathrm{Ca}^{2+}$ levels of WT plants as shown by the $\mathrm{Ca}^{2+}$-selective dye Fluo-3 AM. When leaves of WT and $s k l$ plants were treated after BAW damage with ethephon (Fig. 7b) only the WT leaves showed a significant reduction of cytoplasmic $\mathrm{Ca}^{2+}$ concentrations $\left(\left[\mathrm{Ca}^{2+}\right]_{\text {cyt }}\right)$ which continued to decrease over $20 \mathrm{~min}$. Despite a small initial decrease, the $\left[\mathrm{Ca}^{2+}\right]_{\mathrm{cyt}}$ of $s k l$ was not affected. In line with these observations, pre-treatment with ethephon abolished the rapid increase of $\left[\mathrm{Ca}^{2+}\right]_{\mathrm{cyt}}$ in the WT leaf after BAW damage (Fig. 7c). Conversely, the $s k l$ leaves, which do not respond to ethylene, showed an immediate increase of $\left[\mathrm{Ca}^{2+}\right]_{\text {cyt }}$ in the damaged area.

Altogether, these findings suggest that ethylene can modulate the extent and mode of $\mathrm{Ca}^{2+}$ influx resulting from herbivore attack, either directly or through feedback from ethylene effects on downstream events.

Involvement of $\mathrm{Ca}^{2+}$ signaling in the $\mathrm{BAW}$-induced terpenoid formation

In order to investigate the possible role of $\mathrm{Ca}^{2+}$ signaling on JA and terpenoid biosynthesis, WT plants were treated with the $\mathrm{Ca}^{2+}$-chelator (BAPTA) prior to BAW treatment (Fig. 8). JA (but not 12-oxophytodienoic acid [OPDA], Fig. 8a) levels as well as the levels of DMNT, $\alpha$-copaene, and $\beta$-caryophyllene $(P<0.05 ;$ ANOVA $)$ were reduced (Fig. 8b). These terpenoids represent the final products of MtTPS1, MtTPS3, and MtTPS5 proteins, the genes of which were either or both regulated by JA and JA + ACC (Fig. 4).

\section{Discussion}

The composition of the induced volatile blends depends on plastic phenotypic responses which can be modulated by the interplay of several biosynthetic pathways and levels of signaling molecules (Arimura et al. 2005). In order to dissect the complexity of plastic responses, we used the ethylene-insensitive $s k l$ mutant of $M$. truncatula in combination with defined chemical treatments and BAW exposure. Within the BAW- or treatment $(\mathrm{JA}+\mathrm{ACC})$-induced blends of volatiles, sesqui and homoterpenoids were the most abundant (see Fig. 1a). To identify the genes responsible for terpenoid biosynthesis, we cloned three MtTPS genes, based on sequence similarities with other angiosperm TPS genes, namely MtTPS1, MtTPS3, and MtTPS5. Heterologous protein expression and biochemical analysis of the enzymatic product identified MtTPS1 as a
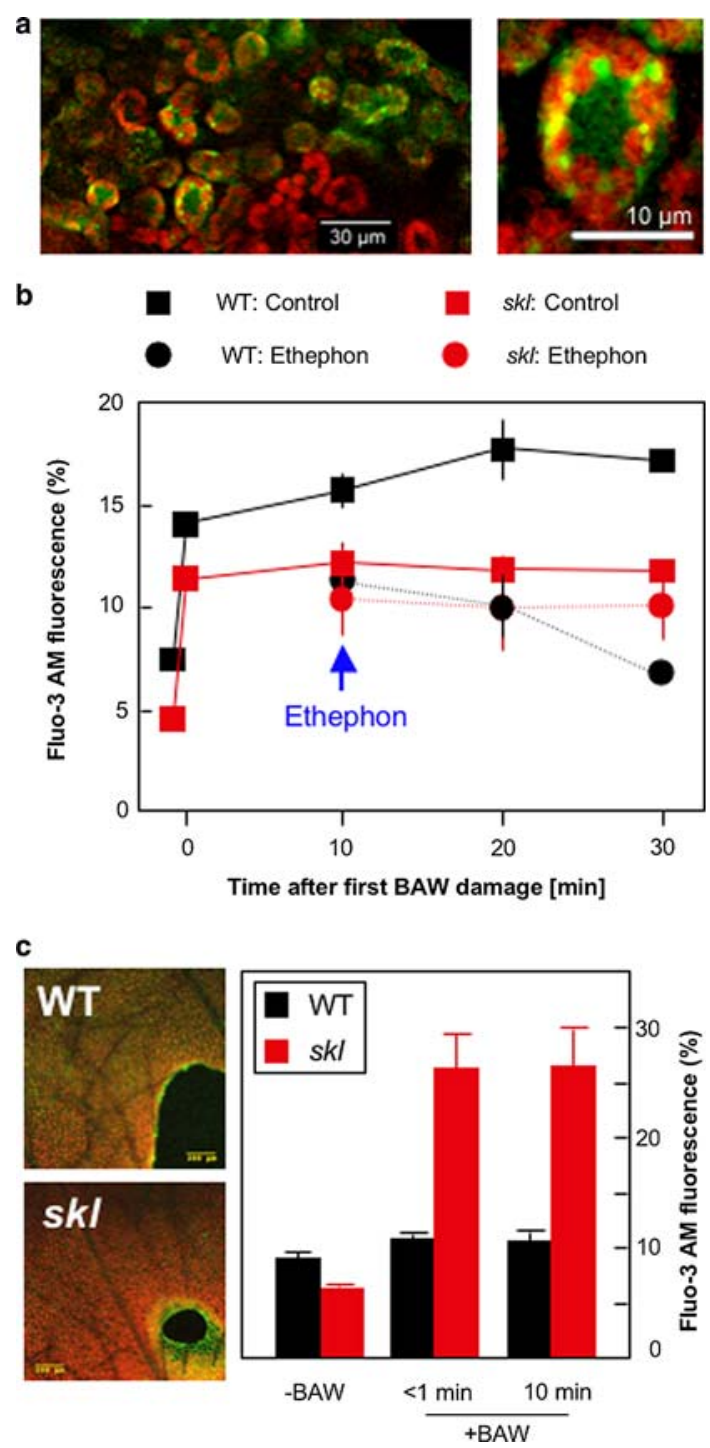

Fig. 7 Effect of ethylene on cytoplasmic $\mathrm{Ca}^{2+}$ concentrations. a A WT leaf was treated with Fluo-3 AM for $1 \mathrm{~h}$ and damaged with a BAW larva. The confocal laser scanning microscope analysis showed false-colour subcellular localization of the dyes, proving that the dyes are loaded mainly into the cytoplasm. The green fluorescence refers to the binding of Fluo-3 AM with $\mathrm{Ca}^{2+}$, whereas the chloroplasts are evidenced by a bright red colour caused by chlorophyll fluorescence. b The dye-loaded WT or $s k l$ leaf was damaged by a BAW larva. After $10 \mathrm{~min}$, the leaf was treated with the ethephon solution $(10 \mathrm{mM})$ or buffer (Control), indicated by a blue arrow. c Alternatively, the dye-loaded WT or $s k l$ leaf was pre-treated with ethephon $(10 \mathrm{mM})$ for $20 \mathrm{~min}$ and exposed to a BAW larva for $10 \mathrm{~min}$. Demonstrated false-colour images show differences between cellular $\mathrm{Ca}^{2+}$ concentrations in WT and $s k l$ leaves $10 \mathrm{~min}$ after ethephon treatment. Data represent the ratio of Fluo-3 AM fluorescence \pm standard deviation (SD) $(n=4)$

$\beta$-caryophyllene synthase, MtTPS3 as a nerolidol/geranyl linalool synthase, and MtTPS5 as a multiproduct sesquiterpene synthase. Multi-product TPSs significantly contribute to the plasticity of blends and are increasingly found in plants, especially in context with herbivory, as shown previously for other 

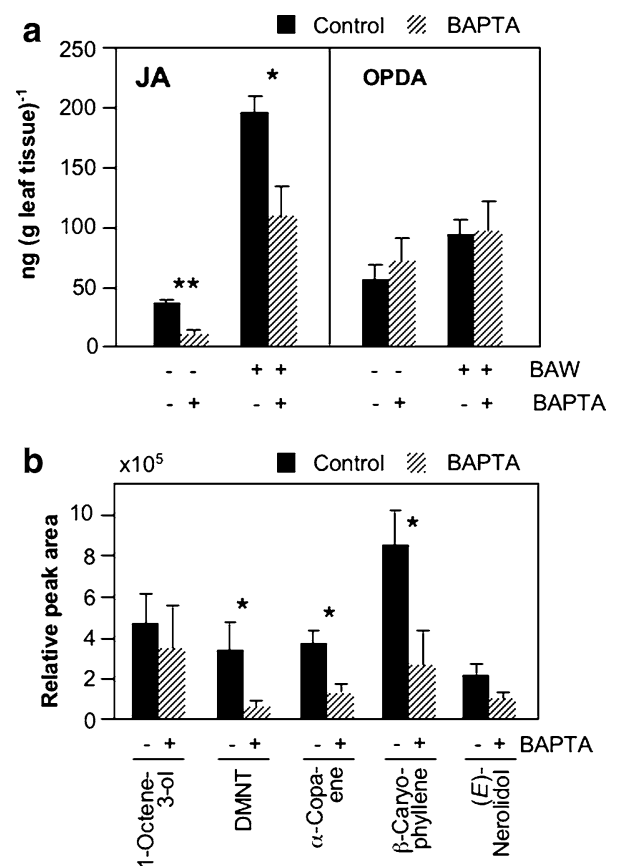

Fig. 8 Biosynthesis of oxylipins (a) and volatiles (b) in WT leaves after treatment with BAPTA or water (control). Leaves to be used for oxylipin analysis were harvested $6 \mathrm{~h}$ after BAW exposure (mean $+\mathrm{SE}$, $n=4)$. Volatiles emitted from the infested plants were collected for $48 \mathrm{~h}$ starting with BAW damage. Emission is presented as relative peak area $(\mathrm{g} \text { shoot tissue })^{-1}$ collected over a 48 -h period $+\mathrm{SE}(n=4)$. $* P<0.05, * * P<0.01$ (ANOVA). Peak areas from integration of the reconstructed ion chromatogram were used without additional calibration

plants (Köllner et al. 2004; Tholl et al. 2005). In addition to olefinic sequiterpenes, the heterologously expressed MtTPS5 protein produced a number of hydroxylated products which were not detected in the gas phase of $M$. truncatula plants (Fig. 1a). Whether these compounds are subsequently modified in planta remains to be clarified.

Conventional pathway allocation suggests that the sesquiterpene precursors, IDP and DMADP, are mainly provided by the cytosolic MVA pathway. However, evidence is emerging that the plastidial MEP pathway may also significantly contribute to cytosolic sesquiterpene biosynthesis by allowing IDP to be shuttled between the different compartments (Piel et al. 1998; Bick and Lange 2003; Bartram et al. 2006). Here we demonstrate this for M. truncatula by the administration of labelled $\left[{ }^{2} \mathrm{H}_{2}\right]$-DOX and pathway-specific inhibitors. These results indicate that the MEP pathway, as well as the cytosolic MEV pathway, plays a dominant role by providing terpenoid precursors.

Compared to WT plants, the $s k l$ mutant produced lower amounts of sequiterpenes and DMNT when challenged with feeding insects, suggesting a possible role for ethylene as a modulator of BAW-induced terpenoid biosynthesis. In fact, ethylene and JA synergistically induce the biosynthesis of certain sesquiterpenes [e.g., $\alpha$-copaene, $(E)$-nerolidol, and germacrene D along with unidentified sesquiterpenes (I)]. The production of these sesquiterpenes coincides with enhanced transcript levels of MtDXS2 and MtTPS5. In contrast, MtTPS1 and MtTPS3 transcripts were only induced by JA treatment.

Feeding BAW raised the expression level of MtTPS3 in WT and $s k l$ plants in the same way, but levels of $(E)$-nerolidol and DMNT formation were higher in WT plants, suggesting that ethylene might have a post-transcriptional impact on the MtTPS3 protein. Another interesting aspect is that MtTPS3 has been claimed to be a plastidial-targeted protein (Gomez et al. 2005), which is also consistent with its ability to produce the diterpene geranyllinalool from GGDP (see Fig. 3b). The subcellular localization of $(E$ )nerolidol formation and subsequent DMNT-production remains unsolved.

Ethylene has been recently shown to enhance sesquiterpenes and suppress monoterpenes in Zea mays (Ruther and Kleier 2005). In this case, the presence of ethylene enhanced the hexenol-induced production of volatiles (linked to increased internal JA levels) by a factor of 5-6 with respect to the sesquiterpenes, but suppressed the constitutively emitted monoterpene linalool (Ruther and Kleier 2005). In M. truncatula the simultaneous application of $\mathrm{JA}+$ ACC also significantly enhanced production of sesquiterpenes. The suppressing effect of ethylene on monoterpene biosynthesis in WT plants becomes obvious from the volatile profile of the $s k l$ mutant: it lacks ethylene perception and as a consequence, constitutively emits limonene. The mode of monoterpene suppression by ethylene in WT $M$. truncatula is still unknown and will require the identification of the limonene synthase and of specific elements that control plastidial monoterpene biosynthesis. It is interesting to note that the sesquiterpene synthases MtTPS1 and MtTPS5 represent multiproduct enzymes that could also synthesize monoterpenes, including limonene. However, since BAW attack enhanced the transcript levels of MtTPSI and MtTPS5 in WT leaves that lacked limonene emission, these enzymes could not be responsible for the production of limonene. The lack of any plastidial transit peptide at the N-terminus of MtTPS1 and MtTPS5 supports their prediction as cytosolic proteins involved in sesquiterpenoid biosynthesis.

WT and $s k l$ plants showed different resting levels of cytosolic $\left[\mathrm{Ca}^{2+}\right]_{\text {cyt }}$; but after BAW feeding, both plants increased at the same rate (see Fig. 7b). Both pre- and posttreatment with ethephon reduced the BAW-triggered $\left[\mathrm{Ca}^{2+}\right]_{\text {cyt }}$ increase in WT leaves, but had no effect on $s k l$ plants. In order to test whether or not this effect is general, we treated the transgenic aequorin-expressing soybean cell culture (Mithöfer et al. 1999) with ethephon and insect regurgitate containing active eliciting compounds. In this artificial system, ethephon abolished the regurgitate-elicited 
and transient increase of $\left[\mathrm{Ca}^{2+}\right]_{\text {cyt }}$ (Fig. S3 of electronic supplementary material). Accordingly, either ethephon or the released ethylene have a direct effect on $\mathrm{Ca}^{2+}$ homeostasis. Depriving plants of extracellular $\mathrm{Ca}^{2+}$ by BAPTA reduces the formation of JA (Fig. 8a) and the release of terpenoids (Fig. 8b). The level of OPDA is not affected. However, the $s k l$ plants only slightly modify the level of $\left[\mathrm{Ca}^{2+}\right]_{\text {cyt }}$ and never completely remove external $\mathrm{Ca}^{2+}$. These facts may explain why the level of JA production after feeding BAW is not significantly different between WT and $s k l$ plants (see Fig. S1 of electronic supplementary material).

Although the simultaneous application of $\mathrm{JA}+\mathrm{ACC}$ strongly stimulates volatile emissions (Fig. 5), this effect may be partly due to the action of ACC, but not of ethylene. ACC, which was found to occur as a conjugate with JA, might act as an additional factor controlling gene expression, similar to the conjugates of JA and isoleucine (Krumm et al. 1995; Staswick and Tiryaki 2004). This assumption is supported by the observation that the combined treatment with JA + ACC + STS does not fully compensate for the effect of JA + ACC (Fig. 5).

In conclusion, our results demonstrate that either the phytohormone ethylene or its precursor (ACC) affects the different levels of BAW-induced terpenoid blends by modulating early signaling events such as the cytoplasmic $\mathrm{Ca}^{2+}$-influx and later the JA-dependent biosynthesis of terpenoids.

Acknowledgments We thank Dr. Giles E. Oldroyd (John Innes Centre) for $s k l$ seeds; Dr. Maritta Kunert and Ms. Anja David for volatile analysis. (-)-Cubebol was generously provided by Dr. Alois Fürstner (Max Planck Institute for Kohlenforschung). This work was supported by the Japanese Society for the Promotion of Science (to G.A.) and the Centre of Excellence CEBIOVEM of the University of Turin (to M.M.).

\section{References}

Aharoni A, Giri AP, Deuerlein S, Griepink F, de Kogel WJ, Verstappen FWA, Verhoeven HA, Jongsma MA, Schwab W, Bouwmeester HJ (2003) Terpenoid metabolism in wild-type and transgenic Arabidopsis plants. Plant Cell 15:2866-2884

Aharoni A, Jongsma MA, Bouwmeester HJ (2005) Volatile science? Metabolic engineering of terpenoids in plants. Trends Plant Sci 10:594-602

Ament K, Van Schie CC, Bouwmeester HJ, Haring MA, Schuurink RC (2006) Induction of a leaf specific geranylgeranyl pyrophosphate synthase and emission of (E,E)-4,8,12-trimethyltrideca-1,3,7,11tetraene in tomato are dependent on both jasmonic acid and salicylic acid signaling pathways. Planta 224:1197-1208

Arimura G, Ozawa R, Shimoda T, Nishioka T, Boland W, Takabayashi J (2000) Herbivory-induced volatiles elicit defence genes in lima bean leaves. Nature 406:512-515

Arimura G, Huber DPW, Bohlmann J (2004a) Forest tent caterpillars (Malacosoma disstria) induce local and systemic diurnal emissions of terpenoid volatiles in hybrid poplar (Populus trichocarpa $\times$ deltoides): cDNA cloning, functional characterization, and patterns of gene expression of (-)-germacrene D synthase, PtdTPS1. Plant J 37:603-616

Arimura G, Ozawa R, Kugimiya S, Takabayashi J, Bohlmann J (2004b) Herbivore-induced defense response in a model legume Two-spotted spider mites induce emission of $(E)-\beta$-ocimene and transcript accumulation of $(E)$ - $\beta$-ocimene synthase in Lotus japonicus. Plant Physiol 135:1976-1983

Arimura G, Kost C, Boland W (2005) Herbivore-induced, indirect plant defences. Biochim Biophys Acta 1734:91-111

Bach TJ, Lichtenthaler HK (1982) Mevinolin: a highly specific inhibitor of microsomal 3-hydroxy-3-methylglutaryl-coenzyme A reductase of radish plants. Z Naturforsch [C] 37:46-50

Bartram S, Jux A, Gleixner G, Boland W (2006) Dynamic pathway allocation in early terpenoid biosynthesis of stress-induced lima bean leaves. Phytochemistry 67:1661-1672

Bergomaz R, Boppré M (1986) A simple instant diet for rearing Arctiidae and other moths. J Lepidopt Soc 40:131-137

Bick JA, Lange M (2003) Metabolic cross talk between cytosolic and plastidial pathways of isoprenoid biosynthesis: unidirectional transport of intermediates across the chloroplast envelope membrane. Arch Biochem Biophys 415:146-154

De Moraes CM, Lewis WJ, Paré PW, Alborn HT, Tumlinson JH (1998) Herbivore-infested plants selectively attract parasitoids. Nature 393:1907-1922

Degenhardt J, Gershenzon J (2000) Demonstration and characterization of $(E)$-nerolidol synthase from maize: a herbivore-inducible terpene synthase participating in $(3 E)$-4,8-dimethyl-1,3,7-nonatriene biosynthesis. Planta 210:815-822

Donath J, Boland W (1994) Biosynthesis of acyclic homoterpenes in higher plants parallels steroid hormone metabolism. J Plant Physiol 143:473-478

Dudareva N, Martin D, Kish CM, Kolosova N, Gorenstein N, Fäldt J, Miller B, Bohlmann J (2003) (E)- $\beta$-Ocimene and myrcene synthase genes of floral scent biosynthesis in snapdragon: function and expression of three terpene synthase genes of a new terpene synthase subfamily. Plant Cell 15:1227-1241

Edelmann HG, Gudi G, Kühnemann F (2002) The gravitropic setpoint angle of dark-grown rye seedlings and the role of ethylene. J Exp Bot 53:1627-1634

Eisenreich W, Bacher A, Arigoni D, Rohdich F (2004) Biosynthesis of isoprenoids via the non-mevalonate pathway. Cell Mol Life Sci 61:1401-1426

Engelberth J, Koch T, Schüler G, Bachmann N, Rechtenbach J, Boland W (2001) Ion channel-forming alamethicin is a potent elicitor of volatile biosynthesis and tendril coiling. Cross talk between jasmonate and salicylate signaling in lima bean. Plant Physiol 125:369-377

Gomez SK, Cox MM, Bede JC, Inoue K, Alborn HT, Tumlinson JH, Korth KL (2005) Lepidopteran herbivory and oral factors induce transcripts encoding novel terpene synthases in Medicago truncatula. Arch Insect Biochem Physiol 58:114-127

Horiuchi J, Arimura G, Ozawa R, Shimoda T, Takabayashi J, Nishioka T (2001) Exogenous ACC enhances volatiles production mediated by jasmonic acid in lima bean leaves. FEBS Lett 509:332336

Jez JM, Ferrer JL, Bowman ME, Dixon RA, Noel JP (2000) Dissection of malonyl-coenzyme A decarboxylation from polyketide formation in the reaction mechanism of a plant polyketide synthase. Biochemistry 39:890-902

Kahl J, Siemens DH, Aerts RJ, Gäbler R, Kühnemann F, Preston CA, Baldwin IT (2000) Herbivore-induced ethylene suppresses a direct defense but not a putative indirect defense against an adapted herbivore. Planta 210:336-342

Köllner TG, Schnee C, Gershenzon J, Degenhardt J (2004) The variability of sesquiterpenes emitted from two Zea mays cultivars is 
controlled by allelic variation of two terpene synthase genes encoding stereoselective multiple product enzymes. Plant Cell 16:1115-1131

Krumm T, Bandemer K, Boland W (1995) Induction of volatile biosynthesis in the Lima bean (Phaseolus lunatus) by leucine- and isoleucine conjugates of 1-oxo- and 1-hydroxyindan-4-carboxylic acid: evidence for amino acid conjugates of jasmonic acid intermediates in the octadecanoid signalling pathway. FEBS Lett 377:523-529

Kuzuyama T, Shimizu T, Takahashi S, Seto H (1998) Fosmidomycin, a specific inhibitor of 1-deoxy-D-xylulose 5-phosphate reductoisomerase in the nonmevalonate pathway for terpenoid biosynthesis. Tetrahedron Lett 39:7913-7916

Leitner M, Boland W, Mithöfer A (2005) Direct and indirect defences induced by piercing-sucking and chewing herbivores in Medicago truncatula. New Phytol 167:597-606

Lücker J, Bouwmeester HJ, Schwab W, Blaas J, van der Plas LHW, Verhoeven HA (2001) Expression of Clarkia S-linalool synthase in transgenic petunia plants results in the accumulation of $S$-linalyl- $\beta$-D-glucopyranoside. Plant J 27:315-324

Maffei M, Bossi S, Spiteller D, Mithöfer A, Boland W (2004) Effects of feeding Spodoptera littoralis on lima bean leaves. I. Membrane potentials, intracellular calcium variations, oral secretions, and regurgitate components. Plant Physiol 134:1752-1762

Maffei ME, Mithöfer A, Arimura G, Uchtenhagen H, Bossi S, Bertea CM, Cucuzza LS, Novero M, Volpe V, Quadro S, Boland W (2006) Effects of feeding Spodoptera littoralis on Lima Bean Leaves. III. Membrane depolarization and involvement of hydrogen peroxide. Plant Physiol 140:1022-1035

McKay SAB, Hunter WL, Godard KA, Wang SX, Martin DM, Bohlmann J, Plant AL (2003) Insect attack and wounding induce traumatic resin duct development and gene expression of (-)-pinene synthase in Sitka spruce. Plant Physiol 133:368-378

Meyer O, Hoeffler JF, Grosdemange-Billiard C, Rohmer M (2004) Practical synthesis of 1-deoxy-D-xylulose and 1-deoxy-D-xylulose 5-phosphate allowing deuterium labelling. Tetrahedron 60:12153-12162

Mithöfer A, Ebel J, Bhagwat AA, Boller T, Neuhaus-Url G (1999) Transgenic aequorin monitors cytosolic calcium transients in soybean cells challenged with $\beta$-glucan or chitin elicitors. Planta 207:566-574

Oldroyd GED, Engstrom EM, Long SR (2001) Ethylene inhibits the Nod factor signal transduction pathway of Medicago truncatula. Plant Cell 13:1835-1849

Ozawa R, Arimura G, Takabayashi J, Shimoda T, Nishioka T (2000) Involvement of jasmonate- and salicylate-related signaling pathways for the production of specific herbivore-induced volatiles in plants. Plant Cell Physiol 41:391-398

Penmetsa RV, Cook DR (1997) A legume ethylene-insensitive mutant hyperinfected by its rhizobial symbiont. Science 275:527-530

Piel J, Donath J, Bandemer K, Boland W (1998) Mevalonate-independent biosynthesis of terpenoid volatiles in plants: induced and constitutive emission of volatiles. Angew Chem Int Ed 37:24782481

Rodríguez-Concepción M, Boronat A (2002) Elucidation of the methylerythritol phosphate pathway for isoprenoid biosynthesis in bacteria and plastids. A metabolic milestone achieved through genomics. Plant Physiol 130:1079-1089

Ruther J, Kleier S (2005) Plant-plant signaling: ethylene synergizes volatile emission in Zea mays induced by exposure to (Z)-3-hexen-1-ol. J Chem Ecol 31:2217-22

Sharon-Asa L, Shalit M, Frydman A, Bar E, Holland D, Or E, Lavi U, Lewinsohn E, Eyal Y (2003) Citrus fruit flavor and aroma biosynthesis: isolation, functional characterization, and developmental regulation of Cstps 1, a key gene in the production of the sesquiterpene aroma compound valencene. Plant J 36:664-674

Schmelz EA, Alborn HT, Engelberth J, Tumlinson JH (2003a) Nitrogen deficiency increases volicitin-induced volatile emission, jasmonic acid accumulation, and ethylene sensitivity in maize. Plant Physiol 133:295-306

Schmelz EA, Alborn HT, Tumlinson JH (2003b) Synergistic interactions between volicitin, jasmonic acid and ethylene mediate insect-induced volatile emission in Zea mays. Physiol Plant 117:403-412

Schulze B, Lauchli R, Sonwa MM, Schmidt A, Boland W (2006) Profiling of structurally labile oxylipins in plants by in situ derivatization with pentafluorobenzyl hydroxylamine. Anal Biochem 348:269-283

Staswick PE, Tiryaki I (2004) The oxylipin signal jasmonic acid is activated by an enzyme that conjugates it to isoleucine in Arabidopsis. Plant Cell 16:2117-2127

Tholl D, Chen F, Petri J, Gershenzon J, Pichersky E (2005) Two sesquiterpene synthases are responsible for the complex mixture of sesquiterpenes emitted from Arabidopsis flowers. Plant J 42:757-771

Veen H (1983) Silver thiosulfate: an experimental tool in plant science. Sci Hortic 20:211-224

Walter MH, Hans J, Strack D (2002) Two distantly related genes encoding 1-deoxy-D-xylulose 5-phosphate synthases: differential regulation in shoots and apocarotenoid-accumulating mycorrhizal roots. Plant J 31:243-54

Wang KLC, Li H, Ecker JR (2002) Ethylene biosynthesis and signaling networks. Plant Cell 14(Suppl):S131-S151 